\title{
Hidrolipodistrofia ginoide: aspectos gerais e metodologias de avaliação da eficácia
}

\author{
Gynoid lipodystrophy: general aspects and methodologies \\ for efficacy evaluation
}

Idalina Maria Nunes Salgado Reis dos Santos', Fernanda Daud Sarruf², Tatiana Santana Balogh', Claudinéia Aparecida Sales de Oliveira Pinto', Telma Mary Kaneko', André Rolim Baby', Maria Valeria Robles Velasco'

\section{Resumo}

A hidrolipodistrofia ginoide (HLDG), popularmente conhecida como celulite, está presente em grande parte da população feminina. Trata-se de uma alteração do relevo cutâneo, envolvendo modificações morfológicas, histoquímicas, bioquímicas e ultraestruturais nos adipócitos, além de alterações na derme e na microcirculação. Existem inúmeras formas de se tratar essa condição, sendo a mais utilizada e de fácil acesso o uso de produtos tópicos contendo princípios ativos com ação vasoprotetora, anti-inflamatória, estimuladores da microcirculação periférica e agentes lipolíticos. Para verificar a eficácia destas formulações, existem metodologias de avaliação envolvendo avaliações instrumentais e clínicas da melhoria do aspecto da celulite. Este artigo teve por objetivo revisar as formas existentes de se avaliar a ação de produtos que atuam na HLDG, considerando os diversos mecanismos de ação, além de revisar aspectos gerais referentes a esta condição.

Palavras-chave: celulite; eficácia; cosméticos; estética; distribuição da gordura corporal.

\begin{abstract}
Gynoid lipodystrophy (HLDG), popularly known as cellulite, is present in a great part of the female population. It is an alteration of the cutaneous relief involving morphological, histochemical, biochemical and ultrastructural modifications in the adipocytes and also alterations in the dermis and microcirculation. There are countless ways of treating this condition, being topic products containing active principles with vessel protective, antiinflammatory actions, peripheral microcirculation stimulators and lipolytic agents the most used and of easiest access. In order to verify the efficacy of these formulations, there are evaluation methodologies involving instrumental and clinical evaluations of the improvement of the aspect of cellulite. Therefore, this article aimed at reviewing the existent ways of evaluating the efficacy of products against gynoid lipodystrophy, taking the different mechanisms of action into account and also at reviewing general aspects concerning this condition.
\end{abstract}

Keywords: cellulitis; efficacy; cosmetics; esthetics; body fat distribution.

Recebido em: 19/7/2010

Revisado em: 12/1/2011

Aprovado em: 14/6/2011

\footnotetext{
Trabalho realizado na Faculdade de Ciências Farmacêuticas da USP e no Instituto de Bioengenharia da Pele (IBP) Evic Brasil Ltda., São Paulo (SP) Brasil. Faculdade de Ciências Farmacêuticas da Universidade de São Paulo (USP) - São Paulo (SP), Brasil.

2 Faculdades Oswaldo Cruz - São Paulo (SP), Brasil.

Endereço para correspondência: Maria Valéria Robles Velasco - Avenida Professor Lineu Prestes, 580, bloco 13 - Cidade Universitária - CEP: 05508-900

- São Paulo (SP) - Brasil - E-mail: mvrobles@usp.br
} 


\section{Introdução}

A hidrolipodistrofia ginoide (HDLG), popularmente conhecida como "celulite", envolve alteração do relevo cutâneo e ocorre em 80 $90 \%$ da população feminina após a puberdade, aparecendo nas regiões da cintura pélvica, membros inferiores e abdômen. Alguns autores consideram-na um atributo próprio do sexo e não um estado patológico ${ }^{1-5}$.

A denominação mais correta para a designação "celulite" é "hidrolipodistrofia ginoide", por ser um termo científico que etimologicamente significa: hidro, de água; lipo, relativo à gordura; distrofia, desordem nas trocas metabólicas do tecido; e ginoide significa forma de mulher. Portanto, a HLDG é uma alteração genuína e locoregional do panículo adiposo subcutâneo determinante do formato corporal característico da mulher, com perda do equilíbrio histofisiológico local ${ }^{6-9}$.

A HLDG modifica a estrutura histológica da pele e altera o tecido conjuntivo e, consequentemente, ocorre polimerização excessiva dos mucopolissacarídeos, o que resulta no aumento da retenção de água, sódio e potássio, conduzindo à elevação da pressão intersticial e gerando compressão de veias, vasos linfáticos e nervos ${ }^{2,8,10-13}$.

O tecido apresenta degeneração das fibras elásticas, proliferação de fibras colagênicas, hipertrofia dos adipócitos e edema ${ }^{8}$. Em função das inúmeras consequências decorrentes da fisiopatologia da HLDG, a abordagem terapêutica deve envolver diversas fontes de ação, incluindo substâncias químicas e equipamentos.

A HLDG pode ser classificada, segundo o aspecto clínico e histopatológico, em quatro graus ${ }^{4,5,14-16}$ :

- Grau I: Assintomático. Sem alterações clínicas observáveis. Na avaliação histopatológica, observa-se: espessamento da camada aureolar, aumento da permeabilidade capilar, anisopoiquilocitose adipocitária, micro-hemorragias diapedéticas, ectasia dos capilares e microaneurismas fusiformes nas vênulas póscapilares;

- Grau II: Apresenta alterações clínicas, como palidez, hipotermia, diminuição da elasticidade, relevo cutâneo altera visivelmente apenas com a compressão ou a contração muscular; sinais visuais mínimos. No exame histopatológico, observa-se: hiperplasia e hipertrofia da trama de fibrilas argentoafins periadipocitárias e pericapilares, dilatação capilar, micro-hemorragias e espessamento da membrana basal dos capilares;

- Grau III: Aspecto de "casca de laranja", sensação palpatória de finas granulações nos planos profundos, dor à palpação, diminuição da elasticidade do tecido, palidez e hipotermia. Na histopatologia, observa-se: dissociação e rarefação do tecido adiposo por fibrilas colágenas neoformadas, com envolvimento encapsulante de pequenas zonas de adipócitos degenerados, formando micronódulos, esclerose e espessamento da camada íntima das pequenas artérias, dilatação das vênulas e pequenas veias, numerosos microaneurismas e hemorragias no tecido adiposo, neoformação de capilares, dissociação do limite dermo-hipodérmico com aumento do volume das papilas adiposas, frequentemente dismórficas e es- clerose do conectivo dérmico, com inclusão de adipócitos no conectivo dos estratos mais profundos da derme.

- Grau IV: Mesmas características do grau III e presença de nódulos palpáveis, visíveis e dolorosos, grandes ondulações na superfície (aspecto de "saco de nozes"). Na histopatologia, observa-se: desaparecimento da estrutura lobular do tecido adiposo e surgem nódulos encapsulados por tecido conjuntivo denso, formação de macronódulos pela confluência de muitos micronódulos, lipoesclerose difusa e importantes alterações da microcirculação, telangectasias, microvarizes, varizes e atrofia da epiderme.

A fisiopatologia da HLDG pode ser dividida em quatro estágios evolutivos $^{14,16}$ :

- A alteração do esfíncter arteriolar pré-capilar conduz à modificação da permeabilidade cápilovenular e ectasia capilar, com transudação e edema pericapilar e interadipocitário;

- O edema dificulta as trocas metabólicas e desencadeia uma resposta conjuntiva, com consequente hiperplasia e hipertrofia do invólucro reticular, resultando na formação de uma trama irregular de fibrilas argentafins (com afinadade à prata) pericapilares e periadipocitárias. Alguns adipócitos, que apresentam anisopoiquilocitose, sofrem laceração, e formam-se vacúolos pluricelulares, rodeados por tramas reticulares de espessura irregular;

- As fibrilas se agregam em fibras colágenas e se distribuem em arranjos capsulares em torno de grupos de adipócitos, formando os micronódulos;

- Ocorre esclerose das traves conjuntivas e formação de macronódulos, pela confluência de muitos micronódulos.

Na histopatologia há três fases evolutivas ${ }^{16}$ :

- Alteração adipocitária, estase linfática e proliferação de fibrócitos;

- Fibroplasia, colagênese e neoformação capilar, micro-hemorragias focais e hiperqueratose folicular, focos de edema discreto na derme. Corresponderia ao aspecto de "casca de laranja".

- Acentuação das alterações anteriores, esclerose do tecido conjuntivo da hipoderme e parte profunda da derme (aspecto acolchoado). A presença de granulação, observada pela palpação profunda da área afetada, corresponde aos nódulos no tecido subcutâneo encontrados no exame histopatológico.

As formas clínicas da celulite podem também ser classificadas como compacta ou dura, flácida, mista ou edematos $a^{10}$ :

- Compacta ou dura: a região atingida, geralmente, possui conformação regular e uniforme, dificilmente apresenta grandes deformações. Mais dolorida e de pior prognóstico, visto que a mobilização do tecido é difícil;

- Flácida: é a forma mais importante, tanto em número quanto nas manifestações aparentes. Segue a forma determinada pela posição 
(deitada, sentada ou em pé), e são comuns as varicosidades associadas a uma sensação de peso nos membros acometidos. Melhor prognóstico;

- Mista: presença de mais de um tipo no mesmo paciente;

- Edematosa: aspecto externo de edema tecidual. Possui consistência variável, que pode vir associada a um linfoedema, os membros apresentam-se em forma de coluna, é de aspecto pastoso e pode ou não apresentar sinal de cacifo, sendo que o prognóstico é sempre ruim.

\section{Aspectos gerais do tratamento da HLDG}

Em função da falta de conhecimentos a respeito dos fatores etiopatogênicos específicos da HLDG, as opções de tratamento devem ter um aspecto abrangente, envolvendo várias técnicas que se complementam ${ }^{5}$.

Apesar da existência de diversas metodologias de tratamento da HLDG, existem poucas evidências científicas da real eficácia, tendo ação limitada ${ }^{5,15}$.

Métodos físicos e mecânicos ${ }^{5,10,15-17}$ :

- Jato de ar térmico: ar sob pressão ${ }^{16}$;

- Aeroidroterapia/hidromassagem: adição de ar sob pressão em banheira de hidromassagem ${ }^{5,10,15-17}$;

- Ultrassom ${ }^{5,10,15-17}$;

- Vacuoterapia (endermólise): a massagem é realizada pela alternância entre sucção e repouso (vácuo) , $10,15-17^{\text {; }}$

- Radiação ultravioleta: promove movimento das partículas de água da região, que colidem com os adipócitos e ocorre sua eliminação $0^{5,10,15-17}$;

- Radiação infravermelha: atua como fototermoterapia ${ }^{5,10,15-17}$;

- Corrente galvânica/iontoforese: ação eletrolítica ${ }^{5,10,15-17}$;

- Corrente farádica: ação sobre a circulação sanguínea e, consequentemente, redução de edema e ativação da secreção glandular ${ }^{5,10,15-17}$;

- Mesoterapia: injeção de substâncias ativas na mesoderme, melhorando a circulação e a metabolização de gorduras, além de estimular a síntese de colágeno ${ }^{18}$;

- Drenagem linfática manual favorece e auxilia o retorno vascular ${ }^{5,16}$;

- Subcision: correção dos defeitos de depressão da pele empregando-se dois mecanismos: o primeiro consiste no corte cirúrgico do tecido fibrótico, que prende os tecidos do sistema músculo aponeurótico à derme; e o segundo, relacionado à formação de tecido conjuntivo recém formado, que se inicia após a resolução do hematoma formado no primeiro ato, no processo de ruptura de alguns vasos ${ }^{19}$;

- Cirurgia plástica: lipectomia (ressecção de blocos de gordura), conhecida como lipoaspiração ${ }^{5,16}$;

- Injeção subcutânea de dióxido de carbono ${ }^{15}$;

- Resfriamento por contato ${ }^{15}$;
- Redução de peso: segundo o estudo realizado por Smalls et al. ${ }^{6}$, em média, a gravidade da celulite diminui com a perda de peso, apesar de, para alguns voluntários do estudo, ter ocorrido efeito contrário.

Dentre todas as formas de tratamento empregadas na HLDG, a mais utilizada e de fácil acesso envolve o uso de produtos cosméticos contendo substâncias ativas, com ação vasoprotetora, anti-inflamatória, estimuladoras da microcirculação periférica e agentes lipolíticos ${ }^{16,20-22}$.

\section{Princípios ativos vasoprotetores}

Envolvem substâncias que melhoram o quadro geral circulatório e linfático ${ }^{8}$. Como exemplos de princípios ativos citam-se:

- Extratos vegetais ricos em flavonoides (pela sua atividade antioxidante);

- Leucoantocianidinas: antirradicais livres e vasoprotetores pertencentes à classe dos flavonoides;

- Saponinas.

\section{Princípios ativos anti-inflamatórios}

Atuam na inibição da síntese de prostaglandinas, leucotrienos, mediadores inflamatórios e inibem a liberação de histamina ${ }^{17}$. Como exemplos de princípios ativos citam-se:

- Extratos vegetais: castanha da Índia, alcaçuz e camomila;

- Ácido glicirrízico e abisabolol;

- Enzimas: mucopolissacaridase ${ }^{17}$.

\section{Estimuladores da microcirculação periférica}

Agem em nível de tecido conjuntivo, regulando a estruturação das formas fibrilares conectivas'. Como exemplos, temos:

- Extratos vegetais estimulantes como: arnica e urtiga;

- Ésteres nicotínicos: nicotinato de metila.

\section{Substâncias de ação lipolítica}

Podem atuar inibindo a fosfodiesterase ou reduzindo o tamanho do adipócito ${ }^{16}$. Ao estimular a lipólise, ocorre seu esvaziamento, melhorando a aparência e a suavidade da superfície da pele. Como exemplos, temos ${ }^{18}$ :

- Bases xantínicas, como cafeína, teofilina e teobromina e o retinol aumentam o AMP cíclico (AMPc), ocorre o estímulo à enzima proteinaquinase, que converterá a enzima triacilglicerollipase em sua forma ativa. A lipase ativada promove a hidrólise dos triacilgliceróis ${ }^{5}$;

- Silícios e iodos orgânicos ${ }^{16}$;

- L-carnitina (transportador de ácidos graxos)? 


\section{Avaliação clínica da eficácia do tratamento da HLDG}

Inúmeras técnicas são conhecidas para a avaliação da eficiência dos produtos. Porém, devido à complexidade, poucas publicações estão disponíveis na literatura abordando a avaliação da eficácia dos tratamentos contra HLDG ${ }^{7,16,23}$.

A HDLG pode ser avaliada em voluntários humanos do sexo feminino, de três diferentes formas ${ }^{16,24,25}$ :

- Os próprios usuários analisam o produto quanto à eficácia por meio de questionários de avaliação subjetiva e qualitativa;

- Os voluntários e um investigador qualificado analisam o produto de forma subjetiva e qualitativa;

- Além do voluntário e do investigador, o produto é também analisado por medidas biofísicas das propriedades biomecânicas da pele, avaliando-se de forma objetiva, quantitativa e mais precisa.

Dentre as técnicas objetivas de análise da eficácia de produtos utilizados no tratamento da HLDG, podemos citar as avaliações: antropométrica, impedância bioelétrica, xerografia, ecografia bidimensional (modo B), termografia de placa, tomografia computadorizada e de ressonância magnética, do relevo cutâneo e anátomopatológica, entre outras.

Devemos considerar que em todas as formas de análise empregadas é importante a prática da validação da metodologia frente a alguns parâmetros analíticos, bem como a calibração periódica dos equipamentos e instrumentos envolvidos e o treinamento da equipe técnica. Convenientemente e de acordo com as necessidades específicas da etapa analítica, devem ser validados os parâmetros a seguir: precisão (dispersão dos resultados para um mesmo analista e entre analistas), exatidão (dispersão dos resultados em relação ao possível valor teórico, que pode ser estipulada frente a um padrão de referência), pesquisa de interferentes de componentes da formulação em relação à substância ativa analisada para métodos de avaliação in vitro, curva de calibração do equipamento e curva analítica (efeito dose-resposta), limites de detecção e/ou quantificação (métodos in vitro).

Para garantir a reprodutibilidade das medidas realizadas, têm-se como requisitos básicos: ambiente controlado (umidade relativa e temperatura), climatização do voluntário e dos equipamentos utilizados, padronização dos procedimentos de medição, protocolos de aplicação das metodologias em condições realísticas, operadores qualificados e uso adequado de prognósticos computadorizados ${ }^{16}$.

\section{Macrofotografia ou documentação fotográfica}

É importante realizar o acompanhamento padronizado da evolução da HLDG por meio de fotografia para verificar a eficácia antes, durante e depois do tratamento utilizado ${ }^{10,15}$.
Esta deve ser obtida em condições padronizadas de iluminação, fundo e posição da câmera e dos voluntários/pacientes em posição reprodutível tanto na foto anterior quanto na posterior ao tratamento, a fim de garantir maior confiabilidade dos resultados ${ }^{7,10}$.

Este procedimento obtém fotografias durante e ao final do tratamento, comparando-as àquelas obtidas antes do seu início, permitindo a avaliação da evolução deste?

Galvão ${ }^{10}$ utilizou este método para avaliação da eficácia da drenagem linfática sobre a celulite de mulheres. Foram obtidas fotos antes e após tratamento, sob condições padronizadas de iluminação, fotógrafo, máquina fotográfica utilizada e posição do paciente. Após a análise das fotos obtidas, foi constatado que não houve melhora significativa no quadro após o tratamento.

A macrofotografia não avalia a HLDG em nível celular, mas caracteriza a aparência da pele típica de celulite. Ao ser combinada com outras técnicas, a macrofotografia pode sim definir a eficácia em reduzir a celulite sob o ponto de vista cosmético?

Bielfeldt et al. ${ }^{7}$ estudaram uma forma de melhorar esta técnica por meio da padronização das condições de obtenção da fotografia. Os autores realizaram um estudo para demonstrar mudanças na celulite visível, em 36 mulheres de 30 a 60 anos com celulite moderada, após uso de um produto anticelulite disponível no mercado durante três meses. As imagens puderam, então, ser classificadas pelos voluntários e por julgadores experientes em relação à severidade da celulite de acordo com uma escala de 10 pontos $(0=$ ausência de celulite; $9=$ mais alto grau de celulite), visto que a variabilidade entre as imagens foi reduzida pela padronização das condições de obtenção destas. Os autores concluíram que o método permitiu a discriminação de melhorias sutis do aspecto da celulite pela utilização de tratamentos cosméticos.

Rossi $^{23}$ realizou um estudo em voluntárias mulheres para verificar a eficácia de um produto contendo um determinado princípio ativo em comparação ao placebo. Nesse estudo, fotografias foram obtidas no mesmo local, antes e após tratamento, com as voluntárias em pé e de costas para a câmera, com os pés unidos estando os glúteos relaxados e contraídos com força máxima.

Recentemente, Hexsel, Dal'Forno e Hexse ${ }^{26}$ desenvolveram e validaram uma escala fotonumérica de avaliação da HLDG, dividida em cinco parâmetros de análise: número de depressões, profundidade das depressões, aparência morfológica das alterações da superfície cutânea, flacidez e grau de celulite. Para todos os parâmetros, foi considerada uma escala de quatro pontos.

Esta técnica é importante pela informação visual de grande impacto e é muito utilizada em congressos científicos da área médica e cosmética, mas devemos ter muito cuidado na padronização da obtenção das imagens.

\section{Avaliações antropométricas}

É o método mais utilizado, em função de sua simplicidade e baixo custo. Trata-se de um método adequado para avaliação da obesidade e 
da adiposidade localizada. Esta metodologia envolve a medição do peso e da altura, utilizados no cálculo do índice de massa corporal (IMC), e das medições da circunferência corporal, empregando uma fita métrica antes e após o tratamento com o produto em teste. O IMC pode ser calculado por meio da Equação 1, $1,16,23,27,28$ :

$$
\text { IMC }=\text { peso } / \operatorname{altura}^{2}(1)
$$

Esta metodologia não reflete significativamente as alterações da HLDG, uma vez que o voluntário pode apresentar perda de peso e, consequentemente, redução das medidas sem, contudo, ter a melhora da $\operatorname{HLDG}^{16,23,27,28}$.

Salgado-Santos ${ }^{16}$ avaliou a eficácia do extrato comercial hidroglicólico de Trichilia catigua e Ptychopetalum olacoides Benthan, com adição do flavonoide rutina, incorporado em formulação cosmética, sobre a melhora do aspecto da celulite em 30 voluntárias de 20 a 40 anos com quadro clínico de HLDG.

A avaliação foi realizada por meio das seguintes metodologias em associação: avaliações antropométricas, ultrassom e avaliação da temperatura cutânea por termômetro infravermelho ${ }^{16}$.

As medidas antropométricas foram realizadas após as voluntárias terem sido submetidas a um período de climatização durante 20 minutos à temperatura de $22 \pm 2^{\circ} \mathrm{C}$ e umidade relativa do ar $55 \pm 5 \%$. Com o estudo, constatouse que o extrato analisado promoveu redução de medidas em determinadas regiões do corpo, indicando eficácia na melhora do referido quadro.

A autora concluiu que a padronização da técnica de medição é possível, permitindo a confiabilidade dos resultados.

Esta técnica foi também empregada por Smalls et al. ${ }^{6}$ como uma das metodologias utilizadas para avaliar a influência da perda de peso sobre o aspecto da celulite em 51 voluntários com celulite visível, comparado com 11 voluntários-controle sem celulite visível e que possuíam peso estável. Neste estudo, avaliou-se a altura, peso e circunferência das coxas.

Smalls ${ }^{4}$ também utilizou este tipo de avaliação para verificar o aspecto da celulite em 51 voluntários após a perda de peso. Foram medidas a altura, peso e circunferência da coxa. Os sujeitos de pesquisa que obtiveram uma diminuição da condição de HLDG apresentaram redução significativa de peso, IMC e circunferência da coxa.

Rossi $^{23}$ realizou as medidas antropométricas em 75 mulheres de 1845 anos com quadro clínico de HLDG, antes e após a utilização de um produto contendo princípio ativo anticelulite em um dos lados (coxas e glúteos) e a utilização do mesmo produto isento de princípios ativos no outro lado. Porém, houve grande variabilidade nos resultados obtidos.

Apesar de ser uma técnica simples, a tomada das medidas exige padronização da técnica de forma a minimizar erros. Adicionalmente, como é reflexo da adiposidade, é interessante ter um acompanhamento nutricional dos voluntários.

\section{Impedância bioelétrica}

Mede-se a resistência aparente de um circuito por meio do fluxo de uma corrente elétrica alternante, empregando eletrodo tetra- polar colocado nas extremidades dos membros superiores e inferiores. Com isso, obtêm-se informações sobre a composição corpórea, dividindo-a em porcentagens de massa magra (músculos, vísceras e ossos), de massa gordurosa (tecido adiposo) e água. Entretanto, este método oferece limitações na avaliação da HLDG, visto que não permite o estudo da microcirculação nem as alterações do tecido conjuntivo $^{16,23,29}$.

\section{Xerografia}

Utiliza-se o elemento selênio eletricamente carregado para irradiar a pele com raios $\mathrm{X}$, ocorrendo a modificação do campo elétrico. A radiação passa através dos tecidos que possuem espessura variável, permitindo a formação de imagens que evidenciam as diferenças de densidade dos tecidos: conjuntivo, muscular e subcutâneo.

Este método possibilita a identificação dos limites da epiderme, derme, tecido subcutâneo e muscular, permitindo a medição de suas espessuras. Por outro lado, trata-se de um método não-inócuo e que não avalia as alterações microcirculatórias ${ }^{23,24}$.

\section{Ensaio de lipólise}

Permite avaliar a capacidade de uma substância estimular ou não a lipólise, empregando um kit específico para este tipo de ensaio ${ }^{18}$.

Caruso et al. ${ }^{18}$ utilizaram com sucesso este tipo de ensaio para avaliar o potencial lipolítico de preparações estimuladoras da lipólise, redutoras de gordura e atenuadoras da aparência da celulite, usadas na prática da mesoterapia cosmética.

O método envolve a utilização de adipócitos humanos diferenciados, dispostos em placas. Estes se aderem à base de cada "poço" da placa tipo wellplate e têm a finalidade de testar os efeitos de vários compostos sobre a lipólise ${ }^{18}$.

As substâncias em teste estimulam ou inibem a liberação de glicerol dos adipócitos, comparado com o emprego da preparação ao tampão (branco). Essa diferença gera o índice: fold induction index ${ }^{18}$.

A placa tipo well plate contém diversos "poços". Em alguns destes, adicionou-se a amostra (substância em teste), em outros o tampão e, em outros, o controle positivo ${ }^{18}$.

Após a adição destas preparações, a placa foi incubada a $37^{\circ} \mathrm{C}$ e $5 \% \mathrm{CO}_{2}$ por 5 horas. Após isso, certa quantidade do conteúdo dos poços foi transferida para uma placa estéril tipo well plate e adicionou-se o reagente de glicerol (reagente do kit do ensaio), para que ocorresse uma reação colorimétrica de intensidade dependente da quantidade de glicerol liberada, a partir de cada substância adicionada nos "poços". A placa foi lida por espectrofotometria a $540 \mathrm{~nm}^{18}$.

A realização de testes in vitro previamente aos testes in vivo é de grande importância. Estes são realizados por ocasião do desenvolvimento de novas substâncias e de formulações dermatológicas/cosméticas como uma triagem na escolha de substâncias, concentrações de uso, 
verificação da compatibilidade entre componentes, a fim de selecionar os resultados de melhor performance.

A discussão a respeito da importância da realização dos testes in vitro, de uma forma geral, é ampla e envolve os segmentos das prescrições manipuladas; formulações industrializadas; pesquisa científica; legalização de uso de produtos; e as publicações científicas no sentido de unir esforços em busca de métodos in vitro que possam se correlacionar cada vez mais com a resposta obtida in vivo.

\section{Ecografia bidimensional (modo B)}

Utilizando transdutores de frequência entre 7,5 e $20 \mathrm{MHz}$, permite a observação da espessura e identificação da presença de nódulos da hipoderme, bem como a determinação do seu diâmetro e da ecotextura do tecido conjuntivo que os envolve $\mathrm{l}^{16,23,30}$. Empregando transdutores de 40 até $200 \mathrm{MHz}$, pode-se visualizar, adicionalmente, a derme papilar e reticular, bem como identificar edema nessa região ${ }^{16,23,30}$.

A ecografia associada ao Doppler permite também avaliar a circulação local ${ }^{16,23,30}$.

Dentre as vantagens deste método, temos o fato de não ser invasivo e capaz de identificar alterações em nível de hipoderme, tecido conjuntivo e circulação ${ }^{16,30}$.

As limitações desta metodologia envolvem a necessidade de equipamento específico e de treinamento técnico especializado (radiologista/dermatologista) $^{16,30}$.

Salgado-Santos ${ }^{16}$ avaliou a variação na espessura da hipoderme decorrente do uso de uma formulação contendo o extrato em teste por meio do aparelho de ultrassonografia, utilizando transdutor linear L12-5 de 2 a 12 MHz de frequência. Segundo a autora, a ultrassonografia modo B indicou ser adequada para avaliar a espessura da hipoderme devido à facilidade de visualização e de medição. Trata-se de uma medida indireta para a avaliação da HLDG. Apesar disso, esta técnica não foi conclusiva no estudo mencionado.

\section{Termografia de placa}

É um método de avaliação da temperatura da superfície da pele, pois na presença da HLDG ocorre sua diminuição ${ }^{16}$.

Colocam-se placas flexíveis compostas por cristais termossensíveis de colesterol em contato com a pele. Após alguns segundos, será originado um "mapa" de cores, de acordo com os diferentes valores de temperaturas na superfície da pele e também a temperatura basal da placa $\left(28 \mathrm{a} 31^{\circ} \mathrm{C}\right)$. De acordo com a imagem termográfica obtida, podese classificar o grau de HLDG (I a IV) ${ }^{16,23}$.

De modo geral, uma imagem termográfica homogênea, uniforme e com áreas escuras hipotérmicas ("buracos negros" e "pele de leopardo") indica grau mais avançado desta condição $0^{14,16,23}$.

Como vantagem desta técnica, citamos que o método é inócuo. Como desvantagens, a exigência de sala com umidade relativa e tem- peratura controladas e constantes. $\mathrm{O}$ resultado pode ser afetado por fatores que ocasionam alterações circulatórias e da temperatura da superfície corporal, tais como a exposição ao sol, fumo, febre, época do ciclo menstrual e estresse, dentre outros. ${ }^{16,23}$

\section{Tomografia computadorizada e de ressonância magnética}

Mede apenas a espessura do tecido adiposo. Portanto, não permitem a avaliação da derme e da microcirculação. São utilizadas principalmente na avaliação da obesidade ${ }^{23,31}$.

\section{Relevo cutâneo}

O relevo cutâneo pode ser avaliado por duas técnicas ${ }^{4,32}$ :

- Réplica de silicone: baseada na transmissão de luz sobre réplica fina de silicone, realizada na região do corpo que se pretende avaliar. Após ter sido colocada em contato na área avaliada, a réplica de silicone é colocada em suporte específico e inserida em um equipamento. Uma fonte de luz deste emite raios paralelos de um lado e a presença de um detector CCD (charged coupled device dispositivo de carga acoplado) do outro lado capta a imagem. A visualização é feita por uma unidade de digitalização e uma placa gráfica conectada a um computador, permitindo observar as ondulações características da HLDG ${ }^{4,32}$;

- Técnica de projeção de franjas: uma luz padronizada é diretamente projetada sobre o local avaliado. Isso permite a obtenção de parâmetros quantitativos e qualitativos relacionados ao relevo cutâneo das áreas do teste, antes e após a aplicação dos produtos ${ }^{4,32}$.

Smalls et al. ${ }^{6}$ quantificaram a severidade da celulite de voluntários antes e após perda de peso em termos de rugosidade da superfície pela técnica de Laser Surface Scanning Tridimensional (parâmetros Sz e Sv). Os autores afirmam que esta técnica permite uma avaliação precisa da percepção humana da severidade da celulite, visto que, em estudos anteriores, esta apresentou correlação com a avaliação subjetiva por voluntários e por julgadores treinados.

\section{Avaliação anátomopatológica}

Consiste em um método invasivo, direto e preciso de avaliação. Pode ser realizado por meio de biópsias no local afetado ${ }^{23,33}$.

Os métodos de coloração empregados nas amostras podem ser: H\&E (hematoxilinaeosina), coloração de rotina na análise histológica; alcian blue, para os mucopolissacarídeos; PAS (ácido periódico e reativo de Schiff, evidenciando as membranas basais); Weigert Van Geison (fucsina resorcina e fuccina ácida), demonstrando as fibras elásticas, colágenas e musculares lisas; tricrômico de Masson (hematoxilina férrica de Weigert, escarlate de Biebrich e azul de ani- 
lina) que fornecem contraste entre as fibras colágenas e musculares da derme $e^{23,34}$.

Smalls ${ }^{4}$ realizou biópsias em pacientes submetidos à cirurgia bariátrica para análise da HLDG, em áreas com e sem celulite. A amostra foi corada com H\&E (para estrutura tecidual), Van Geison (para elastina), tricrômico de Masson (para colágeno) e alcian blue (para proteoglicanos).

Após a biópsia, o material foi submetido à avaliação histológica de adipócitos, profundidade da papila adiposa e infiltração da papila adiposa.

Constatou-se que o tamanho dos adipócitos era significativamente menor em sítios com celulite do que naqueles onde ela está ausente, e observou-se uma diminuição do tamanho dos adipócitos com a perda de peso. Em relação à profundidade da papila adiposa, não foram observadas diferenças entre os sítios. Além disso, verificou-se que a área com celulite possuía significativamente maior infiltração de gordura na papila adiposa.

Rossi $^{23}$ realizou estudo histopatológico em biópsias de pele de mulheres, cujos cortes foram corados com: H\&E, PAS, alcian blue, tricrômio de Masson e Weigert van Gieson. O material (antes e após tratamento) foi avaliado subjetivamente por examinadores e objetivamente por análise de imagem. Por meio deste estudo, foi verificado: espessura da epiderme, espessura da derme papilar, área total da derme, alterações da epiderme, da derme, da microcirculação e do tecido adiposo. A autora observou que as alterações histopatológicas mais frequentes observadas ao microscópio óptico foram o edema da derme e a proliferação das fibras colágenas. Foi também detectada poiquilocitose adipocitária.

\section{Ultrassonografia}

Técnica baseada no fenômeno de interação entre o som e os tecidos, como o subcutâneo e a pele como um todo, isto é, a partir da transmissão do som através do meio, observam-se as propriedades mecânicas dos tecidos ${ }^{35}$.

Os conceitos físicos fundamentais que caracterizam a onda sonora envolvem: comprimento de onda, frequência, período e amplitude. A velocidade do som é constante para cada material e depende tanto das propriedades elásticas como da densidade do meio. Como a maior parte dos elementos constituintes do corpo humano, possui impedâncias acústicas semelhantes, exceto o ar e os ossos, os equipamentos comerciais disponíveis estão calibrados para uma velocidade padrão de $1540 \mathrm{~m} / \mathrm{s}$. Outros conceitos inerentes ao fenômeno de interação som-tecido que devem ser considerados envolvem a impedância acústica (resistência do meio à condição do feixe sonoro) e a absorção da onda ${ }^{25,35}$.

O feixe ultrassônico é gerado por transdutores. As imagens ultrassonográficas são formadas por ecos de curta duração gerados a partir de pulsos rápidos, emitidos pelo equipamento por meio do transdutor ${ }^{25,35}$.

O efeito Doppler, descrito pela primeira vez por Christian Andréas Doppler, em 1841, representa uma forma de quantificar a velocidade do movimento relativo entre uma fonte de um fenômeno periódi- co e um observador. Quando este se distancia da fonte, a frequência da onda sonora ou eletromagnética recebida pelo observador é menor que a efetivamente emitida pela fonte, proporcionalmente à velocidade de deslocamento relativo ${ }^{25,35,36}$.

No caso do ultrassom, a fonte e o observador correspondem ao transdutor. Com isso, são observadas as estruturas refletoras móveis dentro do corpo, notadamente o fluxo sanguíneo, por meio da deteç̧ão do deslocamento das hemácias de frequência da luz espalhada, que geram informações quanto à direção e à velocidade dos fluxos intravascu-

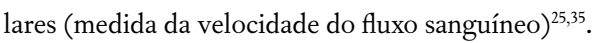

As vantagens desta técnica envolvem: pouca demanda de tempo para sua realização, não invasividade e baixo custo em relação às demais técnicas de imagem ${ }^{37}$.

As principais aplicações práticas da ultrassonografia no estudo da pele envolvem: mensurações da hipoderme e caracterização das lesões, com o dimensionamento e modificação das estruturas cutâneas (como é o caso da HLDG) ${ }^{37}$.

A ultrassonografia cutânea é aplicável tanto para a área de diagnóstico e de acompanhamento dermatológico, quanto para avaliação do efeito (segurança e eficácia) de produtos cosméticos, inclusive daqueles que visam auxiliar o tratamento da HLDG ${ }^{16}$.

Smalls et al. ${ }^{6}$ avaliaram alterações no quadro de HLDG após a perda de peso de voluntários por meio da ultrassonografia tridimensional. A espessura da derme (em milímetros) e a área da superfície da junção entre a derme e tecido subcutâneo (em milímetros quadrados) foram mensuradas utilizando-se o equipamento de ultrassom com uma sonda de $20 \mathrm{MHz}$ (Cortex Technology, Hadsund, Denmark).

Por meio do estudo, os autores concluíram que a densidade de tecido da derme reduziu significativamente com a perda de peso, mas que a espessura da derme e a área de superfície da junção entre a derme e tecido subcutâneo não foram alteradas ${ }^{38}$.

Bielfeldt et al. ${ }^{7}$ utilizaram este mesmo equipamento para avaliar a eficácia de um produto da HLDG, comparando as imagens de ultrassom às da macrofotografia. Apesar de os resultados não indicarem uma melhoria a nível estrutural da celulite, eles descrevem a melhoria na aparência da pele, o que é de grande importância para o consumidor.

Smalls $s^{4}$ utilizou o mesmo equipamento para avaliação da espessura da pele em diversos estudos. A espessura média foi calculada empregando o software do equipamento após a obtenção das imagens de ultrassom colocando-se a sonda verticalmente ao sítio de medida coberto por uma fina camada de gel ${ }^{4}$.

De acordo com o autor, a avaliação por ultrassom foi mais sensível que a observação visual da morfologia da superfície da pele em relação às mudanças na severidade da HLDG (aumento ou redução da celulite) ${ }^{4}$.

\section{Avaliação da temperatura cutânea}

A HLDG promove a diminuição da temperatura da superfície da pele nos locais afetados, o que pode ser detectado empregando termô- 
metros cutâneos apropriados, disponíveis em diversos modelos, sendo os mais conhecidos os de resistência metálica.

Os termômetros cutâneos realizam a leitura da temperatura ao entrar em contato com a pele (equipamentos de contato). Portanto, a medição deve ser imediata, no período de tempo inferior a 2 segundos, de modo a evitar que ocorra aumento da temperatura cutânea por oclusão no manuseio do dispositivo junto à pele ${ }^{36}$.

Os equipamentos como os termômetros de infravermelho capturam a emissividade da pele em comprimentos de onda situados na região a $10500 \mathrm{~nm}$. Estes equipamentos oferecem maior exatidão e podem ser utilizados para verificar as variações da microcirculação cutânea decorrentes de processos inflamatórios, de irritações cutâneas e das próprias variações de temperatura presentes nos quadros de HLDG ${ }^{39}$.

No estudo realizado por Salgado-Santos ${ }^{16}$, foi utilizado um termômetro de infravermelho para avaliação da temperatura cutânea em regiões padronizadas e com celulite, antes e após o tratamento com o produto-teste, para verificação da eficácia deste. As medidas foram efetuadas em quintuplicatas e o produto-teste foi comparado a um controle negativo (formulação isenta de princípios ativos) e a uma referência positiva (mesma formulação adicionada de nicotinato de metila).

A autora considerou o termômetro infravermelho como uma técnica adequada para a avaliação da temperatura cutânea, com as características de sensibilidade, facilidade de utilização, baixo custo, mas exige padronização da técnica de trabalho.

\section{Efeito Doppler na avaliação da microcirculação cutânea}

Como mencionado anteriormente, a HLDG promove a diminuição da temperatura da superfície da pele nos locais afetados. Estudos utilizando Laser-Doppler-Fluxometria indicaram que a média de fluxo sanguíneo nas regiões afetadas pela HLDG é 35\% menor que nas regiões não afetadas ${ }^{40}$.

Por outro lado, a aplicação de produtos cosméticos promove mudança significativa no fluxo sanguíneo da pele. Sendo assim, a quantificação do fluxo sanguíneo pelo monitoramento da microcirculação pode ser uma ferramenta útil para conhecer a segurança e a eficácia das formulações, dentre as quais pode destacar produtos para celulite que visam o aumento da microcirculação ${ }^{40}$.

Podemos citar dois tipos de equipamentos de Laser Doppler: o Probe ou Flowmeter e o Imager ${ }^{36}$.

No primeiro, uma sonda (probe) é posicionada diretamente sobre a pele na região a ser avaliada. O laser transmite luz para a superfície da pele por meio de fibras ópticas que passam através da sonda. A luz transmitida incide sobre as estruturas estacionárias e as hemácias em movimento (da microcirculação) e parte da luz é desviada. Esta última é captada por um detector que fica em contato com a pele e é convertida em sinal elétrico, fornecendo o valor de fluxo. Ao se colocar a sonda sobre a pele para efetuar as medições, acaba ocorrendo restrição da área em função do tempo, e o próprio contato da sonda com a pele pode alterar o valor do fluxo. Além disso, a reprodutibilidade do método não é adequada ${ }^{36}$.

O Laser Doppler Imager é baseado no mesmo princípio que o método anterior, exceto por não ter uma sonda que entra em contato com a pele. O papel das fibras ópticas da sonda é exercido por um conjunto de espelhos e lentes que coletam luz e o espelho se move direcionando o feixe de luz sobre a superfície da pele. Com isso, o equipamento "escaneia" uma determinada área da pele, de maneira padronizada e sequencial. A luz refletida é captada por um detector que não fica em contato com a pele. Após este procedimento, uma imagem colorida é obtida na tela do computador conectado ao equipamento que possui uma escala de cores correspondente aos valores de fluxo da área mensurada ${ }^{36}$.

No estudo realizado por Salgado-Santos ${ }^{16}$, foi utilizada a técnica da ultrassonografia associada ao efeito Doppler para avaliação da eficácia do produto testado na melhoria do quadro da HLDG pela avaliação da microcirculação cutânea.

Inicialmente, as voluntárias foram submetidas a um período de climatização de 20 minutos a $22,0 \pm 2,0^{\circ} \mathrm{C}$ e umidade relativa de $55 \pm 5 \%$. As avaliações por ultrassonografia foram realizadas no antebraço, antes e após a aplicação do produto, comparando-se ao controle negativo e a referência positiva. Os parâmetros avaliados foram: pico sistólico, fluxo sanguíneo e calibre (diâmetro) do vaso. Após a análise dos dados, constatou-se que não houve melhoria na microcirculação com o uso do produto em estudo e a técnica não foi considerada satisfatória para a avaliação da microcirculação cutânea, pela dificuldade de padronização, por ser operador dependente, dispendiosa e sem sensibilidade (baixo comprimento de onda) para avaliar diretamente a vascularização cutânea.

Diversos fatores endógenos exercem influência sobre esta técnica, tais como: medicamentos, idade, raça, estado psicológico, metabolismo, dentre outros; e exógenos, tais como: iluminação sobre a pele, vestuário, condições ambientais de temperatura e umidade, dentre outros. Sendo assim, para assegurar uma melhor confiabilidade torna-se necessário uma boa padronização das condições experimentais para reduzir a ocorrência de vieses ${ }^{16}$.

Segundo Bielfeldt et al. ${ }^{7}$, o aumento do fluxo sanguíneo possui efeito positivo na aparência geral da pele acometida pela HLDG. Os autores utilizaram esta técnica, em associação a outras, para avaliar a eficácia de um produto anticelulite disponível no mercado em voluntários do sexo feminino.

\section{Exame clínico da HLDG}

No exame clínico, é realizada a inspeção, com o paciente ereto e na posição supina, e a palpação (pinçamento da pele entre o polegar e o indicador), visando à observação do aspecto em "casca de laranja”, ondulações, coloração da pele, e modificações associadas como estrias, flacidez, varizes, dentre outras ${ }^{14,16}$.

Durante o exame clínico, avalia-se a elasticidade da pele e a dor ao beliscamento ("pinch test”), além da presença de nódulos na palpação profunda ${ }^{14,16}$. 


\section{Considerações finais}

Existem diversas técnicas de análise de eficácia de produtos empregados na HLDG, mas nenhuma delas encerra todas as informações necessárias para tal avaliação.

Desta forma, atualmente, um conjunto delas deve ser utilizado juntamente com a avaliação clínica para embasar a resposta de um produto na melhoria do quadro da celulite. Sendo assim, existe a tendên- cia de buscar o desenvolvimento de produtos/métodos que atuem na HLDG e de metodologias de avaliação de eficácia cada vez mais inovadores e alternativos, para atender à demanda de um paciente/consumidor cada vez mais consciente e exigente, a fim de oferecer respostas rápidas, não invasivas e próximas da condição real de uso do produto. O tema HLDG ou celulite continuará sendo alvo de pesquisas científicas na área dermatológica/cosmética que busca atender aos desejos dos usuários e alcançar a melhora da qualidade de vida.

\section{Referências}

1. Di Salvo RM. Controlling the appearance of cellulite. Surveying the cellulitereduction effectiveness of xanthines, silanes, CoA, I-carnitine and herbal extracts. Cosmet Toilet Carol Stream. 1995; 110(7):50-9.

2. Kurebayashi AK. HLDG. Cosmet Perf. 2003; 23:156.

3. Rao J, Gold MH, Goldman M. A twocenter, double-blinded, randomized trial testing the tolerability and efficacy of a novel therapeutic agent for cellulite reduction. JCosmet Dermatol. 2005; 4:93-102.

4. Smalls LRK. Development of quantitative models for the investigation of gynoid lipodystrophy (cellulite). Dissertação (Mestrado) - University of Cincinnati, 2005

5. Pavicic T, Borelli C, Korting H C. Cellulite - das größte Hautproblem des Gesunden? Eine Annäherung. JDDG. 2006; 4(10):861-70.

6. Smalls LK, Hicks M, Passeretti D, Gersin K, Kitzmiller, WJ, Bakhsh A, et al. Effect of Weight Loss on Cellulite: Gynoid Lypodystrophy. Plastic and Reconstructive Surgery. 2006; 118(2):510-16.

7. Bielfeldt S, Buttgereit P, Brandt M, Springmann G, Wilhelm KP. Noninvasive evaluation techniques to quantify the efficacy of cosmetic anticellulite products. Skin Research and Technology. 2008; 14:336-46.

8. Gersman A, Tariki MVR, Pinto R. Celulite e gordura localizadas: tratamento com nova formulação de alta eficácia. Rev Cosmiat Med Estét. 1996; 4(3).

9. Ciporkin H, Paschoal LH. Atualização terapêutica e fisiopatogênica da lipodistrofia ginoide. São Paulo: Santos; 1992.

10. Galvão MMM. Drenagem linfática manual e ultrasom no tratamento do fibro edema gelóide em região glútea um estudo de caso. Faculdade Assis Gurgacz, Cascavel: 2005.

11. Barata EAF. A Cosmetologia: Princípios Básicos. São Paulo: Tecnopress; 1995.

12. Sanchez CF. Estudos anatomo-patológicos e termográficos da H.L.D.G. Rev Cosmiat Med Estét. 1994; 2(1):313

13. Curri SB. Cellulite and fatty tissue microciculation. Cosm Toilet. 1993:51-8.

14. Curri SB. Las paniculopatías de estasis venosa: diagnostico clínico e instrumental. $2^{\mathrm{a}}$ ed. Barcelona: Hausmann; 1991.
15. Goldman A, Gotkin RH, Sarnoff DS, Prati C, Rossato F. Cellulite: A New Treatment Approach Combining Subderman Nd: YAG Laser Lipolysis and Autologous Fat Transplantation. Aesthetic Surgery J. 2008; 28(6):656-62.

16. Salgado-Santos IMN. Emprego do ultrasom modo B e com efeito Doppler, termômetro infravermelho e medidas antropométricas na avaliação de uma formulação cosmética anticelulítica contendo extrato hidroglicólico de Trichilia catigua e Ptychopetalum olacoides Bentham. Dissertação (Mestrado) - Faculdade de Ciências Farmacêuticas da Universidade de São Paulo, São Paulo, 2005. p. 183.

17. Magalhães J. Cosmetologia. Rio de Janeiro: Rubio; 2000.

18. Caruso MK, Roberts AT, Bissoon L, Self S, Guillot TS, Greenway FL. An evaluation of mesotherapy solutions for inducing lipolysis and treating cellulite. J Plastic. 2008; 61: 1321-4.

19. Rittes P. Nova técnica terapêutica no tratamento da lipodistrofia ginóide. Rev Cosmiat Med Estét. 1996; 4(3):1826.

20. Tano CTN. Avaliação histológica do tecido adiposo da pele de ratas sob ação da cafeína e Cafeisilane ${ }^{\circledR}$ C. Dissertação (Mestrado) - Faculdade de Ciências Farmacêuticas Universidade de São Paulo, São Paulo, 2003. p. 146

21. Velasco MVR, Tano CTN, Machado-Santelli GM, Consiglieri V0, Kaneko TM, Baby AR. Effects of caffeine and siloxanetriol alginate caffeine, as anticellulite agents, on fatty tissue: histological evaluation. J Cosmet Dermatol. 2008; 7:23-9.

22. Distante F, Bacci PA, Carrera M. Efficacy of a multifunctional plant complex in the treatment of the socalled 'cellulite': clinical and instrumental evaluation. Int J Cosmet Sci. 2006; 28:191-206.

23. Rossi ABR. Lipodistrofia ginóide: aspectos epidemiológicos clínicos histopatológicos e terapêuticos Dissertação (Mestrado) - Faculdade de Ciências Médicas - Universidade Estadual de Campinas, Campinas, 1996. p. 126.

24. Ruvulo E. Avaliação Biofísica da Pele: medida de propriedades físicas e fisiológicas da pele. Cosméticos Online. 1997; 4:41-7.

25. Garcia EAC. Biofísica. São Paulo: Sarvier; 2002.

26. Hexsel DM, Dal'Forno T, Hexsel CL. A validated photonumeric cellulite severity scale. J Europ Acad Dermatol Venereol. 2009;23(5):523-8. 
27. Bray GA, Greenway FL, Molitch ME. Use of anthropometric measures to assess weight loss. Am J Clin Nutr. 1978; 31:769-73.

28. Marshall JDI, Hazlett CB, Spady DW. Quinney, H.A. Comparison of convenient indicators of obesity. Am J Clin Nutr. 1990; 51:22-8.

29. Gray DS, Bray GA, Bauer M, Kaplan K, Gemayel N. Wood R, et al. Skinfold tickness measurement in obese subjects. Am J Clin Nutr. 1990; 51:571-7.

30. Tovo LFR. Contribuição de ultrassonografia no estudo dos tumores cutâneos Dissertação (Mestrado) - Faculdade de Medicina da Universidade de São Paulo, São Paulo, 1994.

31. Seidell JC, Bakker CJG, Kooy K. Imaging techniques for measuring adipose tissue distribution: a comparison between computed tomography and 1.5 T magnetic ressonance. Am Clin Nutr. 1990; 51:953-7.

32. Serup J. Skin irritation: objective chracterization in a clinical perspective. In: Berardesca, E., Elsner, P., Wilhem, K., Maibach, H. Bioengineering of the skin Skin Surface Imaging and Analysis. Boca Raton: CRC Press 1996. p. 26-7

33. Segers AM, Abulafia J, Kriner J., Cortondo 0. "Celulitis": studio histopatológico e histoquímico de 100 casos. Med Cut ILA. 1984; 12 167-72.

34. Lever WF, Lever GS. Histopatologia da pele. $7^{\text {a }}$ ed. São Paulo: Manole; 1991.

35. Cerri GG, Oliveira IRS. Ultrasonografia Abdominal. Rio de Janeiro: Revinter; 2002. p. 130

36. Choi CM, Bennett RG. Laser Dopplers to Determine Cutaneous Blood Flow. Dermatol Surg. 2003; 29(3):272-80.

37. Saito OC, Cerri GG. Ultrasonografia pequenas partes. São Paulo: Sarvier; 1999

38. Ring EFJ. Thermal Imaging of Skin Temperature. In: Serup J, Jemec GBE. Handbook of noninvasive methods and the skin. Boca Raton: CRC Press; 1995; p. 457-71.

39. Welzel J. pH and lons. In: Berardesca E, Elsner P, Wilhem K, Maibach H. Bioengineering of the skin Methods and Instrumentation. Boca Raton: CRC Press; 1995; p. 91-93.

40. Smith WP. Cellulite treatments: snake oils or skin science. Cosm Toil. 1995; 110:61-70. 